\title{
Contents, Vol. 19, 1961
}

Vol. 19

1961

International Archives of

Allergy and Applied

Immunology

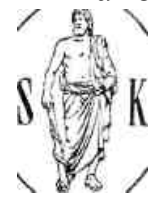

Contributing Editors:

A. v. Albertini

Zurich

D. Bovet

Roma

C.J. G. Britton

London

T. Broman

Göteborg

L. Businco

Roma

A. Cerletti

Basel

H. A. E. v. Dishoeck

Leiden

J. Duchaine

Bruxelles

St. Epstein

Marshfield, Wise.

G. Estrada de la Ríva

Havana

F. J. Farrerons-Co

Barcelona

D. G. R. Findeisen

Dresden

P. Formijne

Amsterdam 
R. A. Good

Minneapolis, Minn.

F. Gröer

Krakow

W. Gronemeyer

Bad Lippspringe

A. Grumbach

Zurich

B. Halpern

Paris

K. Hansen

Heidelberg

E. A. Kabat

Mew York

J. Lecomte

Liige

G. Löfström

Uppsala

J. R. Marrack

Cambridge

R. Meier

Basel

J. A. Moretti

Montevideo

T. Ogata

Tokyo

A. Oliveira-Lima

Rio de Janeiro

D. Ordman

Johannesburg

W. Pagel

London

R. Panzani

Marseille

M. M. Peshkin

New Tork

J. R. Prakken

Amsterdam

W.J. Quarles

van Ufford

Utrecht

A. R. Rich

Baltimore, Md.

B. M. B. Riley

Sidney 


\author{
C. Rimington \\ London \\ M. Rocha e Suva \\ São Paulo \\ B. Rose \\ Montreal \\ G. Ruiz Moreno \\ Buenos Aires \\ J. Runnström \\ Stockholm \\ P. Sangiorgi \\ Milano \\ B. Schick \\ New York \\ H. Schmidt \\ WaberniBern \\ L. Schwartz \\ Washington, D. C. \\ H. Selye \\ Montreal \\ U. Serafini \\ Firenze \\ A. Stoll \\ Basel
}

A. Tiselius

Uppsala

G. L. Waldbott

Detroit, Mich.

Int. Arch. Allergy Printed in Switzerland

Incorporating

Transactions of the Collegium Internationale Allergologicum

Proceedings of the International Association of Asthmology

Proceedings of National Allergy Societies

Founders: P. Kallós, W. Löffler, Fred W. Wittich

Editors-in-Chief:

R. R. A. Coombs, Cambridge

F. Hahn, FreiburgiBr.

D. Harley, London

P. Kallós, Helsingborg

W. Kaufman, Bridgeport, Conn.

Assistant Editor:

G. B. West, London

S. KARGER BASEL - NEW YORK

All rights, including that of translation into foreign languages, reserved.

Photomechanic reproduction (photocopy, microcopy) of this book or part of it without special permission of the publishers is prohibited. 
Copyright 1961 by S. Karger AG, Basel

Printed in Switzerland by Buchdruckerei Aargauer Tagblatt AG, Aarau.

Cliches: Aberegg-Steiner \& Cie., Bern, und Steiner \& Cie. AG, Basel.

Contents - Inhaltsverzeichnis - Sommaire

Vol. 19

Anselmi, B.: vide Sicuteri, F.

Berrens, L. and Young, E.:

Purification and Properties of House Dust Allergen 341

Brun, R. et Musso, E.:

Experiences sur le cobaye sensibilisé aux sulfamido-azo-protéines . . 400

Callaghan, O. Ff.: vide Goldfarb, A. R.

Coombs, R. R. A.; Daniel, M. R.; Gurner, B. W. and Kelus, A.:

Recognition of the Species of Origin of Cells in Culture by Mixed Agglu

tination. II. Use of Heterophile (Anti-Forssman) Sera 210

Cruchaud, A.; Girard, J. P.; Dominé, E. et Micheli, H.:

Etude critique du pouvoir de fixation de Thistamine et de la sérotonine

par le serum sanguin

65

Daniel, M. R.: vide Coombs, R. R. A.

Diamant, M.; Kallós, P. and Rubensohn, G.:

Familial Agammaglobulinemia

193

Diefenbach, H.: vide Winter, $\mathrm{H}$.

Dominé, E.: vide Cruchaud, A.

Freedman, S. O.: vide Perelmutter, L.

Giertz, H. und Hahn, F.:

Weitere Untersuchungen über die Bildung und die Natur des Anaphyla-

toxins 94

Giertz, H.; Hahn, F. und Schmutzler, W.:

Untersuchungen über die Beziehungen zwischen der Intensität der anaphylaktischen Reaktionen des Meerschweinchens und dem Zeitintervall zwischen Erst- und Re-Injektion des Antigens 178

Girard, J. P.: vide Cruchaud, A.

Goldfarb, A. R. and Callaghan, O. H.:

Antigenic Relations between Fractions from Giant and Dwarf Ragweed 86

Gözsy, B. and Kátó, L.:

Factors Other than Histamine Affecting Capillary Permeability .... 168

Gurner, B. W.: vide Coombs, R. R. A.

Hagemeijer, F.: $\quad$ Diagrammes volume/pression du carrefour trachéo-bronchique du cobaye en presence de certains agents pharmacodynamiques 46

Hahn, F.: vide Giertz, H.

Hitzig, W. H.: Zur quantitativen Bestimmung spezifischer Proteinfraktionen. Methodische Untersuchungen mit besonderer Berücksichtigung immunochemi-

scher Methoden. Teil I

145

Teil II 284

Kallós, P.: vide Diamant, M.

Kátó, L.: vide Gözsy, B. 
Keller, R. und Schwarz-Speck, M.:

Zum immunologischen Verhalten isolierter Mastzellen 202

Kelus, A.: vide Coombs, R. R. A.

Korngold, L. and Leeuwen, G. van:

The Use of Cross-Reacting Antiserums for the Study of Antigenic

Heterogeneity of Mammalian y2-Globulins 271

Leeuwen, G. van : vide Korngold, L.

Lind, A.: $\quad$ Serological Studies of Mycobacteria by means of Diffusion- In-Gel Technique.

VI. Physical, chemical and immunological characteristics of precipito-

genic factors in an antigenic preparation from a culture filtrate of a

virulent strain of $\mathrm{M}$. tuberculosis

VII. Investigations of Precipitinogens Demonstrated in Various Prepa

rations of Mycobacterial Origin 112

Micheli, H.: vide Cruchaud, A.

Mueller, A. P. and Wolfe, H. R.:

Precipitin Production Following Massive Injection of BSA in Adult

Chickens 321

Musso, E.: vide Brun, R.

Perelmutter, L.; Sehon, A. H. and Freedman, S. O.:

Fractionation of Sera from Ragweed-Sensitive Individuals by Chromato-

graphy on DEAE-Cellulose 129

Rhodes, J. M.: Chromatography of Tuberculoproteins on DEAE-Cellulose 257

Rubensohn, G.: vide Diamant, M.

Schmutzler, W.: vide Giertz, H.

Schwarz-Speck, M.:

Gel-Diffusionsmethoden bei zwei Allergikern

Schwarz-Speck, M.: vide Keller, R.

Sehon, A. H.: vide Perelmutter, L.

Sicuteri, F.; Testi, A. and Anselmi, B.:

Biochemical Investigations in Headache: Increase in the Hydroxyndole-

acetic Acid Excretion During Migraine Attacks

55

Stender, H.-St. : vide Winter, H.

Stone, S. H.: Acute and Protracted Anaphylactic Shock After Subcutaneous Challenge in Random-Bred and Inbred Guinea-Pigs 227

Strauch, D.: vide Winter, H.

Testi, A.: vide Sicuteri, F.

Tiffeneau, R.: Quantitative Relations Between the Administration of Allergens and the

Production of Bronchoconstrictive Mediators in Allergic Asthma ... 331

Winter, H.; Diefenbach, H.; Strauch, D. und Stender, H.-St. :

Die Antikörperbildung bei der epinephrektomierten Ratte und ihre

unterschiedliche Beeinflussung durch Cortison $\quad 360$

Wolfe, H. R.: vide Mueller, A. P.

Young, E.: vide Berrens, L.

Book Reviews - Buchbesprechungen - Livres Nouveaux $\quad 63,127,192,256,318,377$

New Items - Nachrichten - Nouvelles $\quad 64,254$ 
Obituaries - Nachrufe - Nécrologes 253

Proceedings - Gesellschaftsberichte - Sociétés

Association Internationale d'Asthmologie. 3e Conference Internationale,

Bordighera et Grasse, Octobre 1960

59

Société Française d'Allergie. Seance du 11 février $1961 \quad 250$

British Association of Allergists. Annual General Meeting, London 1961312

Schweizerische Allergie-Gesellschaft - Société Suisse d'Allergie. Jahres-

versammlung, 24. Juni 1961, in Zurich 383 